\title{
Differentiated miRNA expression and validation of signaling pathways in apoE gene knockout mice by cross-verification microarray platform
}

\author{
Hui Han ${ }^{1}$, Yu-Hong Wang ${ }^{1}$, Guang-Jin $\mathrm{Qu}^{1}$, Ting-Ting Sun ${ }^{1}$, Feng-Qing Li ${ }^{1}$, Wei Jiang ${ }^{2}$ and Shan-Shun Luo ${ }^{1}$ \\ The microRNA (miRNA) regulation mechanisms associated with atherosclerosis are largely undocumented. Specific selection \\ and efficient validation of miRNA regulation pathways involved in atherosclerosis development may be better assessed by \\ contemporary microarray platforms applying cross-verification methodology. A screening platform was established using both \\ miRNA and genomic microarrays. Microarray analysis was then simultaneously performed on pooled atherosclerotic aortic \\ tissues from 10 Apolipoprotein E (apoE) knockout mice (apoE - /-) and 10 healthy C57BL/6 (B6) mice. Differentiated miRNAs \\ were screened and cross-verified against an mRNA screen database to explore integrative mRNA-miRNA regulation. Gene \\ set enrichment analysis was conducted to describe the potential pathways regulated by these mRNA-miRNA interactions. \\ High-throughput data analysis of miRNA and genomic microarrays of knockout and healthy control mice revealed 75 \\ differentially expressed miRNAs in apoE $-I-$ mice at a threshold value of 2 . The six miRNAs with the greatest differentiation \\ expression were confirmed by real-time quantitative reverse-transcription PCR (qRT-PCR) in atherosclerotic tissues. \\ Significantly enriched pathways, such as the type 2 diabetes mellitus pathway, were observed by a gene-set enrichment \\ analysis. The enriched molecular pathways were confirmed through qRT-PCR evaluation by observing the presence of \\ suppressor of cytokine signaling 3 (SOCS3) and SOCS3-related miRNAs, miR-30a, miR-30e and miR-19b. Cross-verified high- \\ throughput microarrays are optimally accurate and effective screening methods for miRNA regulation profiles associated with \\ atherosclerosis. The identified SOCS3 pathway is a potentially valuable target for future development of targeted miRNA \\ therapies to control atherosclerosis development and progression. \\ Experimental \& Molecular Medicine (2013) 45, e13; doi:10.1038/emm.2013.31; published online 8 March 2013
}

Keywords: apolipoprotein E knockout mice; atherosclerosis; genome microarray; miRNA microarray; SOCS pathway

\section{INTRODUCTION}

Arteriosclerotic vascular disease (ASVD), commonly known as atherosclerosis, is a chronic inflammatory disease that affects both large and medium arteries. The disease is characterized by the involvement of numerous genes as well as varied environmental stimuli. Using gene-targeted or transgenic mice models, contemporary researchers have confirmed the roles of more than 80 individual genes in atherosclerosis progression and development. ${ }^{1}$ Apolipoprotein E (apoE) has a central role in lipoprotein metabolism, where it is required for the efficient clearance of chylomicrons and very-low-density lipoproteins. Because of the unique role of apoE, Mice lacking apoE (apoE-/-) are well-established models, often used in studying lipoprotein metabolism and atherosclerosis. ${ }^{2}$
MicroRNAs (miRNAs) are small, noncoding RNAs with lengths of approximately 22 nucleotides. These molecules have critical roles in the regulation of many diverse biological pathways, including those associated with various pathologies. ${ }^{3,4}$ To date, over 1000 differentially expressed miRNAs in the human genome, only a small fraction of existent miRNAs, have been characterized and cataloged in the miRNA database (http://www.mirbase.org/). In developing tissues, miRNAs are differentially expressed at high dynamic ranges, and this variation in miRNA expression has been etiologically linked with numerous pathological conditions. ${ }^{5-7}$ The expression levels of at least $30 \%$ of all genes, including genes critical during differentiation processes in developing mammalian cells, are regulated by miRNAs. Notably, the expression levels

\footnotetext{
${ }^{1}$ Department of Gerontology, The First Hospital of Harbin Medical University, Harbin, China and 2Department of Biomathematics, College of Bioinformatics Science and Technology, Harbin Medical University, Harbin, China

Correspondence: Dr S-S Luo, Department of Gerontology, The First Hospital of Harbin Medical University, Harbin 150001, China.

E-mail: Luoss63@126.com

or Professor W Jiang, College of Bioinformatics Science and Technology, Harbin Medical University, Harbin 150081, China.

E-mail: bioccjw@126.com
}

Received 3 July 2012; accepted 7 December 2012 
observed are highly specific to certain tissues, organs or cytotypes. ${ }^{8,9}$

Differential miRNA expression in clinical cardiovascular disease has been previously demonstrated in humans, ${ }^{10}$ where the most compelling evidence of miRNAs in endothelial cells originated as a byproduct of angiogenesis research. For instance, miRNA type miR-92a has been shown to inhibit angiogenesis, whereas miRNA type miR-126 has been shown to sustain vascular integrity and promote angiogenic signaling. ${ }^{7}$ Furthermore, localized miRNA expression profiles that may have a notable role in pathological occurrences vary dramatically between tissues of the heart and arteries, ${ }^{6}$ an observation that has recently been leveraged in research pertaining to vascular inflammation. In addition, the variant miRNA expression profiles observed in oxidized low-density lipoprotein (LDL)-stimulated human primary peripheral blood monocytes further confirms the role of these molecules in cardiovascular-related conditions. ${ }^{5}$ In rat models, variant miRNA expression profiles have been associated with the development of neointimal lesions of the carotid artery, a condition with distinctly different characteristics than related intimal hyperplasia processes. ${ }^{7}$ The recent elucidation of the mechanism of miR-10a regulation in proinflammatory phenotypes of prelesion atherosusceptible endothelium both in vivo and in vitro provides additional support for the importance of miRNAs in the management of arterial pathologies. ${ }^{11}$

Contemporary research has shown that miRNAs may have a more important role in disease onset and progression than originally suspected. Various miRNAs have been implicated in human diseases, such as cancer, ${ }^{12-14}$ developmental abnormalities, ${ }^{15}$ muscular diseases ${ }^{16}$ and cardiovascular disorders. ${ }^{10,17}$ Only recently, however, has the importance of miRNAs in inflammatory diseases been recognized and characterized. ${ }^{18,19}$ Previous research has associated subtle variations in miRNA expression levels with numerous cardiovascular conditions. The seemingly ubiquitous involvement of miRNAs in cardiovascular pathologies suggests that a correlation likely exists between the expression of certain undetermined miRNAs and ASVD.

On the basis of these findings, atherosclerosis likely results in a specific pattern of miRNA and target mRNA expression. This hypothesis was evaluated by examining the miRNA regulation profile associated with atherosclerotic aortic plaques using simultaneous screening and cross-verification of apoE gene knockout (apoE -/-) mice and healthy C57BL/6 (B6) mice. Selective validation of miRNAs and enrichment of the interrelated molecular pathways associated with screened miRNAs and mRNAs provides a detailed regulatory profile of miRNAs in atherosclerosis development that are potentially useful for the development of new targeted therapies.

\section{MATERIALS AND METHODS}

\section{Animal subjects}

Ten male apoE - / - mice were constructed from mice of the C57BL/6 (B6) gene background (apoE-/-B6). Ten healthy, male control mice (B6) were obtained from the Experimental Animal Center at Peking University Health Science Center (Beijing, China). Mice were supplied with standard food containing $6.5 \%$ fat, $19.5 \%$ protein and no cholesterol (Keao Xieli Feed, Beijing, China), as well as untreated tap water ad libitum throughout the study. All food was sterilized by irradiation $\left({ }^{60} \mathrm{Co} \gamma\right)$ before feeding (Keao Xieli Feed). All mice were killed at the age of 8 months. The study was approved by the Ethical Committee of Harbin Medical University, and was conducted in adherence to all applicable state and university guidelines.

\section{Aortic tissue isolation and staining}

At 8 months of age, all mice were anesthetized with sodium pentobarbital (Nembutal, $75 \mathrm{mg} \mathrm{kg}^{-1}$, intraperitoneally), and phosphate-buffered saline containing $5 \mathrm{Uml}^{-1}$ of heparin was poured through the left ventricle of the heart. Under a stereomicroscope (Olympus SZX-ZB7; Olympus, Tokyo, Japan), the aorta was carefully excised. Aortic segments were immediately stored in liquid nitrogen for further analysis.

Hematoxylin and eosin staining was used to visualize tissue samples. Briefly, proximal aorta samples were prepared by careful dissection and followed by continuous fixation in $4 \%$ paraformaldehyde in $0.1 \mathrm{moll}^{-1}$ phosphate buffer ( $\mathrm{pH} 7.4$ ) for $24 \mathrm{~h}$. Samples were then immersed for $15 \mathrm{~min}$ in a buffer solution containing $10 \%$ sucrose, and for $30 \mathrm{~min}$ in a buffer containing $20 \%$ sucrose. Samples were then embedded in optical coherence tomography compound (Tissue-Tek; Milestone Laboratories, Elkhart, IN, USA) and snapfrozen. Transverse sections with $10 \mu \mathrm{m}$ thicknesses were stained with hematoxylin and eosin and Oil Red $\mathrm{O}$ for further microscopic examination.

\section{Total RNA isolation}

Total RNA from each aortic segment was collected using TRIzol (Invitrogen, Carlsbad, CA, USA) and an miRNeasy Mini Kit (Qiagen, Hilden, Germany) according to the manufacturer's instructions. Following RNA quantification using the NanoDrop 1000 (ThermoFisher Scientific, Wilmington, DE, USA), equal amounts of RNA from healthy aortic wall samples of apoE $-/$ - and $\mathrm{B} 6$ mice were divided into the atherosclerotic group (A) and normal group $(\mathrm{N})$.

\section{Microarray and analysis of miRNA}

A and $\mathrm{N}$ samples were labeled using the miRCURY Hy3/Hy5 Power Labeling Kit (Exiqon, Vedbaek, Denmark) and hybridized on the miRCURY LNA Array v.16.0 (Exiqon). After washing, slides were scanned using the Axon GenePix 4000B microarray scanner (Axon Instruments, Foster City, CA, USA). Scanned images were then imported into GenePix Pro 6.0 software (Axon) for grid alignment and data extraction. Replicated miRNAs were averaged, and miRNAs with intensities $>50$ in all samples were used in the calculation of a normalization factor. Expression data were normalized using the median normalization value. After normalization, differentially expressed miRNAs were identified through fold-change filtering.

\section{Microarray of mRNAs}

The RNA integrity of A and $\mathrm{N}$ samples was then assessed by standard denaturing agarose gel electrophoresis. Approximately $5 \mu \mathrm{g}$ of the total RNA from each sample was used for labeling and array hybridization. Reverse transcription was completed using a Superscript double stranded cDNA Synthesis Kit (Invitrogen) followed by double stranded cDNA labeling using a NimbleGen One-color DNA Labeling Kit (Roche Diagnostics GmbH, Mannheim, Germany), 
according to the manufacturer's instructions. Array hybridization was performed using the NimbleGen Hybridization System (Roche Diagnostics $\mathrm{GmbH}$ ) followed by washing with a NimbleGen Wash Buffer Kit (Roche Diagnostics $\mathrm{GmbH}$ ). Finally, array scanning using the Axon GenePix 4000B microarray scanner (Molecular Devices Corporation, Sunnyvale, CA, USA) was conducted, resulting in the acquisition of scanned images in high-resolution tagged image file format. These images were subsequently imported into NimbleScan software v.2.5 (Roche Diagnostics $\mathrm{GmbH}$ ) for grid alignment and analysis of expression data.

\section{Bioinformatic analysis}

Expression data were normalized through quantile normalization using the Robust Multichip Average algorithm included in the NimbleScan software package. Gene level files were imported into Agilent GeneSpring GX software v.11.5.1 (Agilent Technologies, Santa Clara, CA, USA) for further analysis. Genes with values greater than or equal to 100 (all targets value) in the $\mathrm{A}$ and $\mathrm{N}$ sample groups were selected for further analysis. Differentially expressed genes were identified through fold-change filtering.

\section{Quantitative reverse-transcription PCR (qRT-PCR)}

TaqMan MicroRNA Assays (Applied Biosystems, Warrington, UK) were used for the quantitative analysis of miRNA expression. Monitoring of miRNA-derived PCR products was performed on an ABI7500 quantitative PCR system (Applied Biosystems). Quantitative analysis of mRNA levels was conducted by SYBR Premix Ex Taq II (Perfect Real Time; Takara-Bio, Ohtsu, Japan). U6 RNA and $\beta$-actin were used as endogenous internal controls to normalize the expression levels of miRNAs and mRNAs, respectively. Total RNAs were converted to cDNAs using PrimeScript reverse transcriptase (TakaraBio). Species-specific primers were designed as follows:

Suppressor of cytokine signaling-3 (SOCS3) forward primers: $5^{\prime}$-AGAAGATTCCGCTGGTACTG-3' and backward primer: $5^{\prime}$-GCTG GGTCACTTTCTCATAGG-3'; $\beta$-actin forward primer: $5^{\prime}$-TTGTGCG TGACATCAAAGAG- $3^{\prime}$ and backward primer $5^{\prime}$-ATGCCAGAAGATTCCATACC- $3^{\prime}$.

\section{Western blot}

Following extraction of the aorta tissues, SDS-polyacrylamide gel electrophoresis and western blot analysis were performed as previously described. ${ }^{20}$ Briefly, the extracted aortic tissues were prepared by homogenization in lysis buffer at $4{ }^{\circ} \mathrm{C}$, followed by centrifugation at $20000 \mathrm{~g}$ at $4{ }^{\circ} \mathrm{C}$ for $10 \mathrm{~min}$ to remove debris. The resultant supernatants were subjected to western blotting. Briefly, lysates were separated in SDS-polyacrylamide gel electrophoresis gels and transferred to Sequi-Blot polyvinylidene fluoride membranes (BioRad, Hercules, CA, USA). Blots were then incubated at $4{ }^{\circ} \mathrm{C}$ overnight with diluted primary antibodies (anti-SOCS3 1:1000), as recommended in the manufacturer's instructions, followed by incubation with horseradish peroxidase-conjugated secondary antibodies. Signals were detected using Immobilon reagent (Millipore, Billerica, MA, USA) and visualized using an LAS-400 Lumino image analyzer (Fujifilm, Tokyo, Japan). Visualized signal intensities were quantitatively analyzed using Multigauge software (Fujifilm, Japan). Primary antibodies for anti-SOCS3 were purchased from Abcam Biotechnology (Cambridge, MA, USA). An antibody for $\beta$-actin was purchased from Sigma-Aldrich (St Louis, MO, USA).

\section{miRNA-target prediction}

miRNA-target interactions were predicted using 11 established miRNA target-prediction programs: DIANA-microT, MicroInspector, miRanda, MirTarget2, miTarget, NBmiRTar, PicTar, PITA, RNA22, RNAhybrid and TargetScan. ${ }^{6}$ In order to improve the reliability of the miRNA targets, only target genes predicted by at least four programs were selected for extraction.

\section{Functional enrichment analysis}

Functional enrichment analysis was performed through previously developed hypergeometric testing methods using the Kyoto Encyclopedia of Genes and Genomes, designed to investigate the potential regulation of various pathways by differentially expressed miRNAs. ${ }^{5}$ Briefly, this process involved the construction of a refined gene set for differentially expressed miRNAs capable of satisfying the following three criteria:

(1) Genes were predicted target genes for differentially expressed miRNAs.

(2) Differential expression was observed in atherosclerosis.

(3) Differential expression patterns were negatively correlated to those of differentially expressed miRNAs.

Next, the extent of appearance of each gene in the refined gene sets of the Kyoto Encyclopedia of Genes and Genomes pathways was calculated according to the method previously described in the DAVID Bioinformation Database. ${ }^{11}$

\section{Statistical analyses}

All data were expressed as means \pm s.d. Analysis of variance tests or Student's $t$-tests were used for statistical analyses. All miRNAs were considered to have significant differential expression if they were up- or downregulated by at least twofold. Statistical significance was determined when $P$-value was less than $0.05(<0.05)$.

\section{RESULTS}

\section{Confirmation of the apoE $-/$ - model}

Examination of lesion morphology in the proximal aorta transverse sections in apoE and C57BL/6J mice revealed clear formation of artherosclerotic plaques (Figures la and b). The foam cells of the plaque were apparent in Oil Red O-stained samples (Figure 1c), demonstrating the validity of the model. Notable, no phenotypic variations were observed in the apoE - / - model group.

\section{Differential expression of miRNAs between B6 and apoE - / - mice aortic walls}

Following normalization, the average values for each miRNA cluster were used for statistical analysis. A total of 75 miRNAs were differentially expressed in the apoE - / - mouse aorta samples, of which 30 demonstrated upregulation. Among these upregulated miRNAs, the most dramatic differentiation expressions were observed for mmu-miR-34a (37.8-fold increase), mmu-miR-465c-5p (21.7-fold increase) and mmumiR-9* (21.3-fold increase; Table 1). Among the other 45 downregulated miRNAs, the largest decreases in expression were observed in mmu-miR-30e (25-fold decrease), mmumiR-29a (16.7-fold decrease) and mmu-miR-669n (12.5-fold 

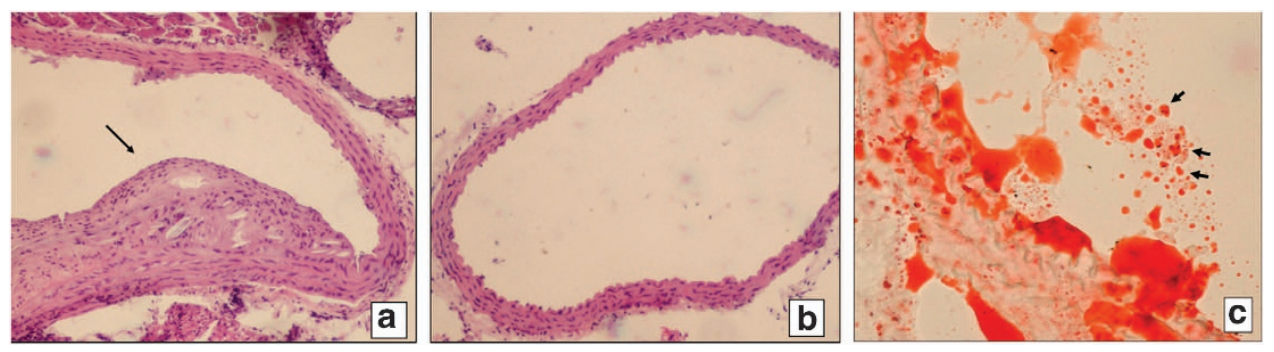

Figure 1 Immunohistochemical examinations of proximal aorta transverse sections (a) Apolipoprotein E (ApoE) knockout mice stained with hematoxylin and eosin $(\times 100)$ (arrow indicates the atherosclerotic plaque), (b) C57BL/6J mice stained with hematoxylin and eosin $(\times 100)$ and $(c)$ ApoE knockout mice proximal aorta transverse sections stained with Oil Red 0 ( $\times 400$; arrows indicate the foam cells of the atherosclerotic plaque).

decrease) (Table 2). On the basis of the differential expressions observed in these miRNAs, a heat map was generated to indicate distinguishable miRNA expression profiles between apoE $-/-$ and normal B6 aortic tissues (Figure 2).

\section{Validation of microarray results using qRT-PCR}

Microarray results were validated by qRT-PCR analysis of apoE $-1-$ and B6 aortic tissue microarray results for miRNA types mmu-miR-34a, mmu-miR-465c-5p, mmu-miR-9*, mmu-miR-30e, mmu-miR-29a and mmu-miR-669n. RNA samples from all apoE - / - and B6 samples were pooled for this analysis. In affected tissues, the expression levels of mmumiR-34a, mmu-miR-465c-5p and mmu-miR-9* were upregulated, whereas the expression levels of mmu-miR-30e, mmumiR-29a and mmu-miR-669n were downregulated. All the six examined miRNAs demonstrated comparable expression levels by both microarray and qRT-PCR analyses (Figure 3). These findings further confirm that atherosclerosis-related miRNAs may participate in ASVD.

\section{Differential gene expression between $\mathrm{B} 6$ and apoE $-I-$ aortic tissues}

The appearance of a twofold (200\%) change in filtering was used as the criteria for differential expression. Of the 44170 gene sequences evaluated, differential expression levels in B6 and apoE $-/-$ aortic tissue arrays were observed in 2283 genes. Among these genes, 1480 genes demonstrated increased expression in apoE $-/-$ mice relative to healthy controls (upregulation $>200 \%$ ), and 803 genes demonstrated decreased expression in apoE $-/-$ mice relative to healthy controls (downregulation by $>50 \%$ ).

\section{Functional enrichment analysis for differentially expressed miRNAs}

A total of 400 genes were identified as part of the refined gene set, each serving as a target gene for a specific miRNA. In atherosclerotic apoE $-/-$ aortic tissues, 75 differentially expressed miRNAs that exhibited a negative correlation with regulated miRNAs were identified. Using these genes, significantly enriched type 2 diabetes mellitus pathways $(P<0.01)$ were identified. The miRNAs regulating the refined genes in
Table 1 Upregulation of miRNAs in atherosclerotic tissue

\begin{tabular}{|c|c|c|}
\hline Accession number & Name & Fold change $(A / N)$ \\
\hline MI0000584 & mmu-miR-34a & 35.8 \\
\hline MIMAT0004873 & mmu-miR-465c-5p & 21.7 \\
\hline MIMAT0000143 & mmu-miR-9* & 11.3 \\
\hline MIMAT0004572 & mmu-miR-290-3p & 11.1 \\
\hline MI0001524 & mmu-miR-431 & 5.6 \\
\hline MIMAT0000527 & mmu-miR-16 & 4.9 \\
\hline MIMAT0016996 & mmu-miR-185* & 3.7 \\
\hline MIMAT0003783 & mmu-miR-615-3p & 3.6 \\
\hline MI0004516 & mmu-miR-763 & 3.6 \\
\hline MI0000569 & mmu-miR-21 & 3.4 \\
\hline MI0000233 & mmu-miR-191 & 3.0 \\
\hline MIMAT0004525 & mmu-miR-99b* & 2.8 \\
\hline MIMAT0017330 & mmu-miR-669e* & 2.8 \\
\hline MIMAT0004790 & mmu-miR-503* & 2.7 \\
\hline MIMAT0003725 & mmu-miR-675-5p & 2.7 \\
\hline MI0000792 & mmu-miR-375 & 2.7 \\
\hline MIMAT0004524 & mmu-miR-30b* & 2.6 \\
\hline MIMAT0004850 & mmu-miR-883b-5p & 2.5 \\
\hline MIMAT0004524 & mmu-miR-669d* & 2.4 \\
\hline MI0004689 & mmu-miR-705 & 2.3 \\
\hline MI0004134 & mmu-miR-668 & 2.3 \\
\hline MI0004695 & mmu-miR-711 & 2.3 \\
\hline MIMAT0017235 & mmu-miR-758* & 2.3 \\
\hline MIMAT0017234 & mmu-miR-592* & 2.2 \\
\hline MI0009949 & mmu-miR-1954 & 2.2 \\
\hline MIMAT0004871 & mmu-miR-465b-5p & 2.2 \\
\hline MIMAT0005292 & mmu-miR-582-3p & 2.1 \\
\hline MIMAT0017015 & mmu-let-7a-2* & 2.1 \\
\hline MI0000698 & mmu-miR-214 & 2.0 \\
\hline MI0003538 & mmu-miR-503 & 2.0 \\
\hline
\end{tabular}

Abbreviation: miRNA, microRNA.

Note: Accession number, miRNA no, in miRBase; Name, alphanumerical call sign for miRNA; Fold change (A/N), miRNA expression ratio of atherosclerotic/ normal.

this pathway exhibited an inverse expression pattern when compared with the refined genes of the pathway shown in Table 3. The miRNA-mRNA interaction network associated with type 2 diabetes pathway is further detailed in Figure 4. 
Table 2 Downregulation of miRNAs in atherosclerotic tissue

\begin{tabular}{|c|c|c|}
\hline Accession number & Name & Fold change (N/A) \\
\hline MI0000259 & mmu-miR-30e & -25.0 \\
\hline MI0000576 & mmu-miR-29a & -16.7 \\
\hline MI0009951 & mmu-miR-669n & -12.5 \\
\hline MIMAT0004825 & mmu-miR-423-5p & -10.0 \\
\hline MIMAT0000670 & mmu-miR-222 & -8.3 \\
\hline MI0000221 & mmu-miR-10a & -8.3 \\
\hline MIMAT0004536 & mmu-miR-151-5p & -7.8 \\
\hline MIMAT0000136 & mmu-miR-125b-5p & -7.1 \\
\hline MIMAT0000210 & mmu-miR-181a & -7.1 \\
\hline MI0006301 & mmu-miR-467g & -7.1 \\
\hline MI0000405 & mmu-let-7d & -6.7 \\
\hline MI0000571 & mmu-miR-23a & -6.7 \\
\hline MI0014047 & mmu-miR-3084 & -6.7 \\
\hline MI0000235 & mmu-miR-193 & -5.6 \\
\hline MIMAT0000521 & mmu-let-7a & -5.6 \\
\hline MI0000144 & mmu-miR-30a & -4.2 \\
\hline MI0000137 & mmu-let-7g & -4.0 \\
\hline MI0004693 & mmu-miR-709 & -3.8 \\
\hline MI0006293 & mmu-miR-467f & -3.7 \\
\hline MI0000407 & mmu-miR-106b & -3.7 \\
\hline MIMAT0000137 & mmu-miR-126-5p & -3.7 \\
\hline MI0000564 & mmu-miR-15a & -3.4 \\
\hline MIMAT0000711 & mmu-miR-365 & -3.4 \\
\hline MI0004690 & mmu-miR-706 & -3.3 \\
\hline MI0000156 & mmu-miR-130a & -3.1 \\
\hline MI0000257 & mmu-miR-143 & -3.0 \\
\hline MI0000575 & mmu-miR-26b & -3.0 \\
\hline MI0000695 & mmu-miR-210 & -2.9 \\
\hline MIMAT0000513 & mmu-miR-19b & -2.8 \\
\hline MI0000558 & mmu-let-7b & -2.7 \\
\hline MI0000138 & mmu-let-7i & -2.6 \\
\hline MIMAT0000138 & mmu-miR-126-3p & -2.6 \\
\hline MIMAT0017323 & mmu-miR-669k* & -2.6 \\
\hline MI0000169 & mmu-miR-145 & -2.6 \\
\hline MIMAT0000523 & mmu-let-7c & -2.6 \\
\hline MIMAT0017255 & mmu-miR-491* & -2.5 \\
\hline MI0000237 & mmu-miR-195 & -2.5 \\
\hline MI0000174 & mmu-miR-152 & -2.4 \\
\hline MIMAT0000536 & mmu-miR-29c & -2.4 \\
\hline MI0000146 & mmu-miR-99a & -2.4 \\
\hline MIMAT0017002 & mmu-miR-204* & -2.4 \\
\hline MIMAT0003780 & mmu-miR-490-3p & -2.3 \\
\hline MIMAT0000546 & mmu-miR-103 & -2.3 \\
\hline MI0000570 & mmu-miR-22 & -2.2 \\
\hline MI0004673 & mmu-miR-669c & -2.0 \\
\hline
\end{tabular}

Abbreviation: miRNA, microRNA.

Note: Accession number, miRNA no. in miRBase; Name, alphanumerical call sign for miRNA; Fold change (N/A), miRNA expression ratio of normal/ atherosclerotic.

mRNA and protein expression of SOCS3 and SOCS3-related miRNAs in individual tissues

According to the interaction network revealed to be associated with the type 2 diabetes pathway (Figure 4), the miRNAs mmu-miR-30a, mmu-miR-30e and mmu-miR-19b have a functional role in indirectly regulating SOCS3 in ASVD.
The expression levels of these miRNAs in seven individual samples from each group were further analyzed by qRT-PCR, demonstrating that mmu-miR-30a, mmu-miR-30e and mmu-miR-19b expression levels were significantly lower in apoE $-/$ - aortic tissues than the levels observed in healthy controls $(P<0.01$; Figure 5$)$. In addition, the SOCS3 mRNA and protein expression levels in each group were further analyzed by qRT-PCR and western blot. As a result, SOCS3 expression levels were shown to be significantly higher in apoE - / - aortic tissues than the levels observed healthy controls (Figures 6 and 7).

\section{DISCUSSION}

Six specific miRNAs associated with ASVD were identified using high-throughput miRNA microarray analysis. These miRNAs may provide novel biomarkers for arthrosclerosis disease progression useful in development of future targeted therapies. When coupled with contemporary miRNA-target prediction programs and gene expression profiles (mRNA), miRNA profiling offers a unique new strategy for addressing ASVD development and progression in both research and clinical settings. The initial step in realization of the immense clinical potential of the miRNAs associated with atherosclerotic regulation is the full characterization of these miRNAs and their affected pathways, a process that began in the current study.

Comparison of miRNA levels observed in atherosclerotic and normal aortic tissues of the mouse ASVD model provides useful information on miRNA regulation in atherosclerosis. The earliest mouse models of atherosclerosis, developed by Wissler in the 1960s, were diet-induced. ${ }^{21}$ These models were inaccurate because the diet was shown to be in itself inflammatory in mice, inducing hepatic nuclear factor- $\kappa \mathrm{B}$ activation and the expression of acute-phase reactants associated with arthrosclerosis. ${ }^{22}$ In 1992, apoE $-/-$ mice were first generated by inactivating the $A p o E$ gene by targeting. ${ }^{23}$ Notably, the apoE $-/-$ mouse used in the current study was the first model developed that showed severe and rapid development of atherosclerotic plaques without dietary intervention. ${ }^{24,25}$ In genetically homozygous mice, this model limits the errors in dietary models caused by very small lesion size and confinement of lesions to the aortic root, often resulting in failure to develop beyond early foam-cell, fatty-streak stages. ${ }^{21}$ The homozygous apoE - / mice used in the current study exhibited only a single phenotype pertinent to the study of atherogenesis, resulting in similar body weight and health, but distinct lipid and lipoprotein profiles compared with normal mice of the same species. Previous studies have established the use of the apoEdeficient models apoE $-/-$ and nlrp3-/ -, apoE $-/-$ and asc $-/-$, and apoE $-/-$ and caspase-1-/ - demonstrating negligible phenotypical variation in the parameters associated with arthrosclerosis, including disease progression, infiltration of plaques by macrophages and plaque stability in all the apoE - / - models. Notably, the same study also revealed four variant genotypes through smooth muscle actin staining. ${ }^{26}$ 


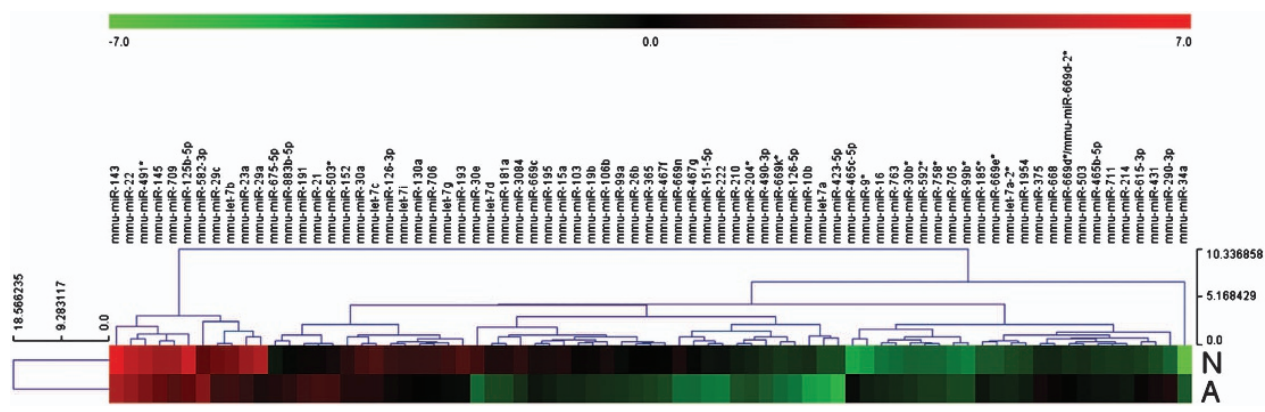

Figure 2 Heat map of the expression profiles of differentially expressed microRNAs (miRNAs).

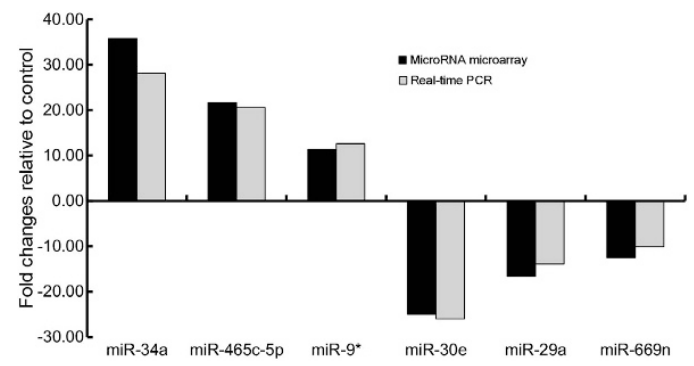

Figure 3 Relative expression levels of six selected microRNAs (miRNAs) in atherosclerotic and healthy aortic tissues analyzed by miRNA microarray and TaqMan quantitative reverse-transcription PCR (qRT-PCR). The six miRNAs with the highest fold changes are listed on the horizontal ordinate. The vertical ordinate refers to the fold changes of relative expression levels between atherosclerotic and healthy aortic tissues. Black bars indicate the microarray results, and gray bars indicate the qRT-PCR results.

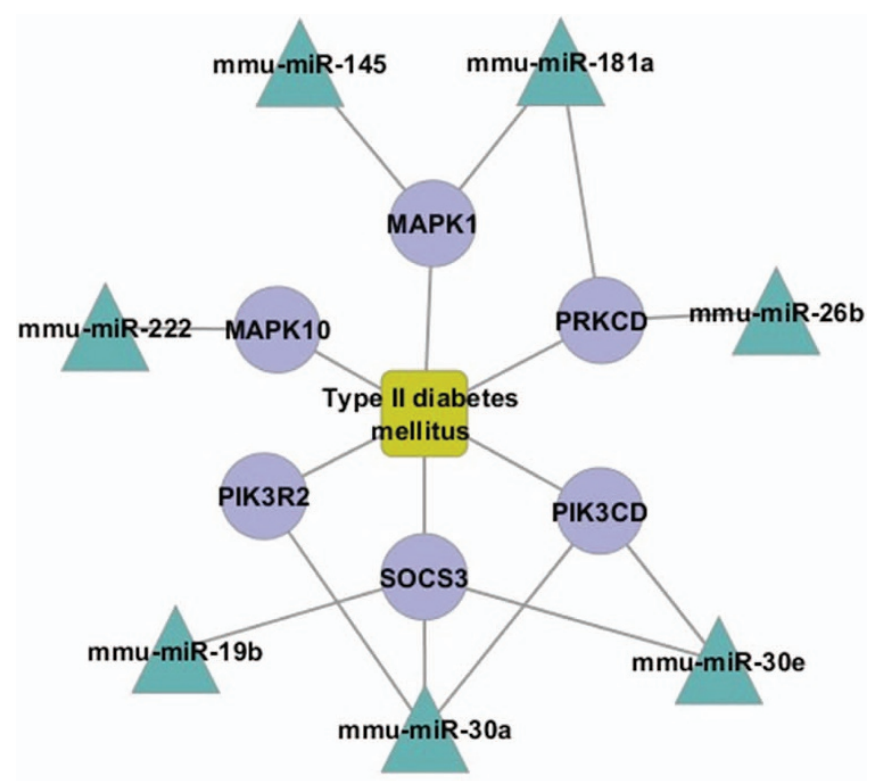

Figure 4 The microRNAs (miRNA)-mRNA interaction network associated with the type 2 diabetes pathway is shown. Round regions represent the genes of the refined gene set. The triangular region represents miRNAs that negatively regulate the refined genes. SOCS3, suppressor of cytokine signaling-3; MAPK10, mitogenactivated protein kinase-10; PRKCD, protein kinase C, delta; PIK3CD, phosphoinositide-3-kinase, catalytic, delta polypeptide; PIK3R2, phosphoinositide-3-kinase, regulatory subunit 2.
Although apoE - / - mice have been shown to be genetically inclined to hypercholesterolemia on high-fat diets and interleukin- $1 \alpha$, not interleukin-1 $\beta$, most significantly contributes to atherosclerosis pathogenesis, these genotypic variations are not significantly expressed in the phenotype of apoE $-/-$ mice. $^{26,27}$ Thus, the apoE $-/-$ mice in the present study were phenotypically equivalent in terms of artherosclerotic features.

In the current study, differential expression was observed in 75 of 1891 examined miRNAs by comparing atherosclerotic and normal aortic tissues using an miRNA microarray platform. Of these, 45 miRNAs were significantly downregulated and 30 were upregulated in atherosclerotic aortic tissues, indicating the significant involvement of miRNAs in ASVD tissues. Furthermore, the current study was also able to confirm previous findings that miR-34a and miR-21 are highly upregulated in atherosclerotic plaques compared with the levels observed in healthy arteries. ${ }^{28}$ The upregulation of miR-21, miR-214, miR-9 and miR-99b coupled with the downregulation of miR-423-5p, miR-15a, miR-143 and miR145 expression shown in the present study also confirms the previously reported findings of Chen et al. ${ }^{5}$ in a study of oxidized LDL-stimulated human primary monocytes. ${ }^{10}$ The downregulation of miR-10a in apoE - / - aortic tissues is also consistent with previous observations of atherosusceptible arterial endothelial tissues in swine, in which notable up- and downregulation of certain miRNAs was observed. ${ }^{7}$

Although exploration of the breadth of miRNAs impacting pathological conditions like ASVD has only recently begun, contemporary data suggests that miR-155 has a distinct expression profile. In fact, it may have a crucial role in various physiological and pathological processes, such as hematopoietic lineage differentiation, immunity, inflammation, cancer and cardiovascular disease. ${ }^{29}$ It has also been reported that miR-155 silencing enhances both the inflammatory response and lipid uptake in oxidized LDL-stimulated human THP-1 macrophages. ${ }^{30}$ Results of the current study show, however, that miR-155 is slightly increased in atherosclerotic tissues compared with control tissues. This increase occurs at a rate 1.31-fold higher than in normal tissues, confirming the previously speculative association between this miRNA and ASVD. Furthermore, Chen et al. ${ }^{5}$ previously reported that miR-125a-5p serves as a partial regulator of the inflammatory response, lipid uptake and oxidized LDL-stimulated 
Table 3 Significantly enriched KEGG pathways in the refined gene set

\begin{tabular}{lcc}
\hline Significant pathways & Refined pathway genes & Negatively regulated miRNAs \\
\hline Type II diabetes mellitus & MAPK1, SOCS3, PIK3CD, MAPK10, & mmu-miR-181a, mmu-miR-145, mmu-miR-19b, \\
ID: mmu04930 & PRKCD, PIK3R2 (six genes) & mmu-miR-30a, mmu-miR-30e, mmu-miR-222, \\
& & mmu-miR-26b (7 miRNAs) \\
\hline
\end{tabular}

Abbreviation: KEGG: Kyoto Encyclopedia of Genes and Genomes.

The miRNAs that regulate the refined genes in the type 2 diabetes pathway show an inverse expression pattern compared with the refined genes in the pathway shown in Table 3; P-values are a result of the enrichment analysis. Note: The significant KEGG pathways in which the refined gene set was enriched are shown.

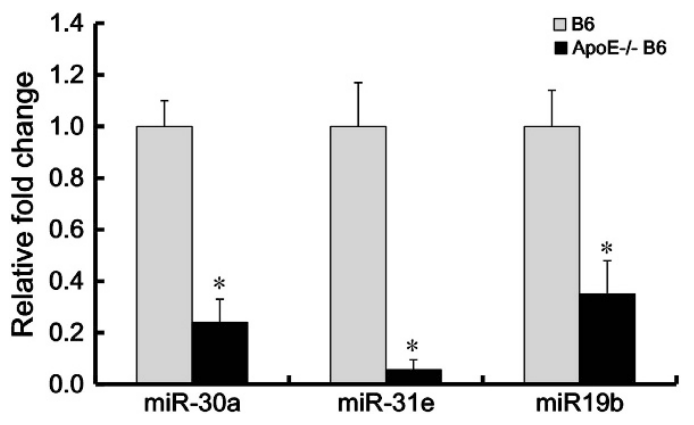

Figure 5 Expression of miR-30a, miR-30e and miR-19b in atherosclerotic and normal aortic tissues were evaluated by TaqMan quantitative reverse-transcription PCR (qRT-PCR), revealing downregulation of these microRNAs (miRNAs) in apolipoprotein $E$ knockout (apoE -/-) aortic tissues compared with levels observed in B6 control aortic tissues. The expression values for the B6 controls were set at 1 . Data are indicated as means \pm s.d. of seven individual subjects; ${ }^{*} P<0.05$ versus $\mathrm{B} 6$ controls.

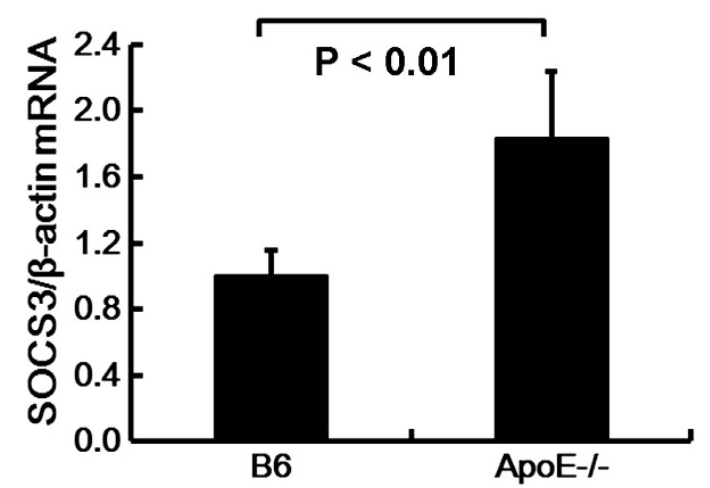

Figure 6 Expression of suppressor of cytokine signaling-3 (SOCS3) mRNA in atherosclerotic (apolipoprotein E knockout (apoE-/-)) and control (B6) aortic tissues is shown. The expression values for the B6 controls were set at 1. Data are indicated as the means \pm s.d. of seven individual subjects; $P<0.01$ versus $B 6$ controls.

monocyte/macrophages, along with other miRNAs and proteins. Surprisingly, the current study revealed that miR$125 a-5 p$ expression was slightly reduced in atherosclerotic tissues, exhibiting a presence in effected tissues that was $20 \%$ lower than that observed in normal tissues. Although mammalian cells have many similarities, this particular discrepancy may be because of variations in tissue type or cell species examined in these studies. a
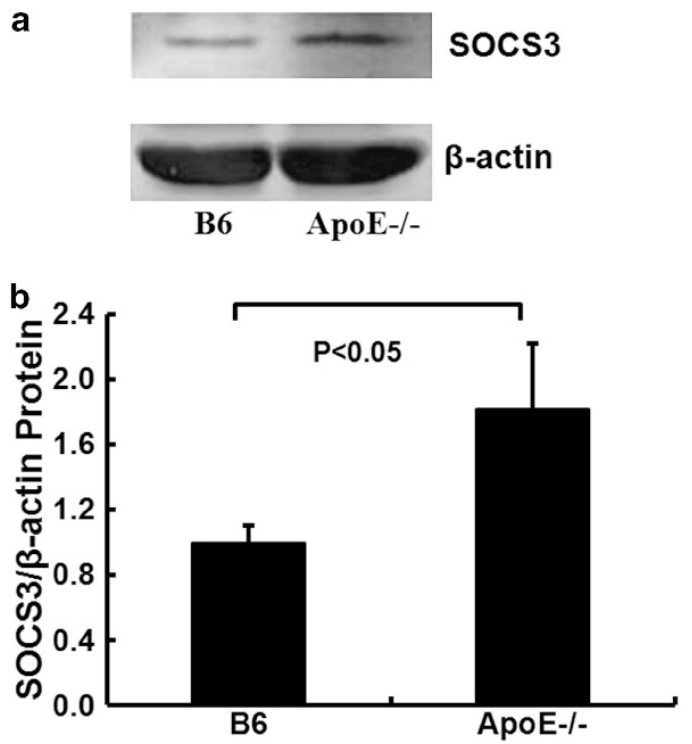

Figure 7 (a) The expression of suppressor of cytokine signaling-3 (SOCS3) proteins extracted from aortic tissues and examined by western blotting. (b) Intensities of the bands corresponding to SOCS3 and $\beta$-actin (a) were determined densitometrically. The intensity values of SOCS3 normalized to $\beta$-actin are shown. Notably, protein samples obtained from two independent experiments were subjected to western blot analysis, demonstrating similar results.

The potential for variation in miRNA expression is particularly high when examining aortic tissues due to the many cytotypes found in the aorta, including endothelial cells, macrophages, smooth muscle cells and fibroblasts. Each of these cytotypes also adds significant complexity to the regulatory progress, making full characterization of these compounds difficult to elucidate. Novel miRNA-mediated regulatory mechanisms associated with ASVD and lipoprotein metabolism have been determined by miRNA profiling coupled with functional testing. It should be noted, however, that simple steady-state expression profiling of miRNAs and mRNAs present several limitations, including an inability to observe active Ago-complexed miRNAs or repressed mRNAs. Thus, future studies are required to determine the precise components and mechanisms responsible for miRNA expression level variations in human ASVD before therapeutic use is possible. Notably, the study of animal models provides an effective means for perfecting miRNA isolation methods and 
generating useful potential biomarkers, many of which may appear analogously in humans and other mammalian cells.

By analyzing predicted miRNA targets using miRecords, 400 differentially expressed target genes were identified as negatively regulated by the 75 miRNAs isolated in the present study. The Kyoto Encyclopedia of Genes and Genomes pathway enrichment analysis identified enriched type 2 diabetes mellitus pathways associated with ASVD, findings that are concurrent with recent studies. The current findings also support the previously observed association between type 2 diabetes mellitus and ASVD. ${ }^{31}$ In diabetic patients, signal transduction in atherosclerosis increase the risk of developing ASVD. ${ }^{32-34}$ Similarly, insulin has a key role in the pathogenesis of both type 2 diabetes mellitus and ASVD, ${ }^{33}$ as exhibited by the upregulation of SOCS3 and downregulation of the SOCS3 negative-mediator miRNAs (miR-19b, miR-30a and miR-30e) currently observed in ASVD tissues. This phenomenon may also be observed in the enriched type 2 diabetes mellitus pathway.

Numerous processes involved in arthrosclerosis are governed by miRNAs. During arthrosclerosis, macrophages engulfed with cholesterol undergo a complete gene profile and phenotypic conversion to foam cells. ${ }^{5}$ The miRNAs miR125a-5p, miR-146a, miR-146b-5p, miR-155 and miR-9 have all been shown to be significantly upregulated in response to oxidized LDL during this process. Thus, vascular cellular proliferation and inflammation involving these cytotypes are likely atherosclerotic processes governed by miRNAs. Other processes, such as calcification, plaque vulnerability and thrombosis may also be affected, though full investigation of these mechanisms has not yet been conducted. ${ }^{35}$ Significantly lower expressions of miR-19b, miR-30a and miR-30e were observed in atherosclerotic tissues than in healthy control tissues using qRT-PCR (Figure 5), findings consistent with previous reports showing that miR-30a inhibits processes of invasion and metastasis, ${ }^{32}$ that the miR-30 gene family has a central role in adipocyte development ${ }^{34}$ and that miR-19b inhibits angiogenesis by blocking cell cycle progression in endothelial cells. ${ }^{36}$ Further studies will be required to prove that these miRNAs are, in fact, associated with the predicted target gene SOCS3. The current experimental results, however, suggest that this association is likely through evidence that the SOCS3 gene mRNA and proteins were increased in atherosclerotic tissues (Figures 6 and 7). Furthermore, previous findings of increased SOCS3 expression in aortic lesions of apoE - / - mice ${ }^{37,38}$ and associations with atherosclerosis development ${ }^{39}$ were supported by the present results. Thus, miRNAs are likely to have a role in the development of atherosclerosis via regulation of SOCS3 or interactions with SOCS3-related pathways.

The SOCS pathway is an endogenous anti-inflammatory, anti-apoptotic pathway closely related to ASVD development. It acts as a negative regulator of the JAK/signal transducer and activator of transcription (STAT) signaling pathway that functions in the stress response signaling pathway involved in cell proliferation, apoptosis and differentiation. The SOCS pathway regulates these processes in many different cytotypes, including vascular endothelial cells, smooth muscle cells and macrophages. It also participates in the formation of atherosclerotic plaques. JAK/STAT-associated genes have been shown to modify granulopoiesis and neutrophil immune functions through the SOCS, and STAT pathways. Moreover, activation of the JAK/STAT pathways may sometimes rescue neutrophils from apoptosis. By inhibiting the JAK/STAT signaling pathway, SOCS proteins can reduce the expression of inflammatory factors and apoptotic genes. Another important ability of SOCS proteins is inhibition of cell proliferation or apoptosis, thereby maintaining the dynamic equilibrium required for normal cell function and activity. Aside from the three identified SOCS3-related miRNAs, other ASVD-related miRNAs may also be involved in the regulation of ASVD. The discovery of specific miRNA involvement in SOCS regulation suggests that it may be possible to develop therapeutics that regulate SOCS expression and inhibit the cytokine signaling pathway. Such techniques may provide a clinically viable method for reducing the development and progression of ASVD in human patients in the future.

The current findings provide a comprehensive characterization of miRNA expression in ASVD; however, detailed information pertaining to miRNAs and their target functions in specific cell types remains to be elucidated in a more controlled setting. As numerous cytotypes are present in aortic tissues, often exhibiting very different miRNA expression levels, it is important to note that the current sampling methods may have affected the outcomes of the present study. These samples were taken from the arterial intima and inner media layer, enabling the conclusion that this miRNA profile is representative of tissue changes in the diseased artery itself instead of merely the intimal plaque. These results, however, must be interpreted with caution, as the contributions of processes in the outer media and adventitia cannot be completely precluded by current methods, necessitating methods with greater control for future work. In addition, these results should be validated by further examination of immunohistochemical characteristics and lesion morphology. Previously, apoE - / - mice have been shown to have abnormal $\mathrm{T}$ lymphocytes levels ${ }^{40}$ and differences in antibody affinities ${ }^{41}$ compared with normal mice. Although lymphocytes have not been reported in atherosclerotic lesions in apoE $-/-$ mice models, the B lymphocytes CD4 + , CD8 + and CD23 + have been demonstrated in aortic fatty streaks in vasculitisprone MRL/lpr mice. ${ }^{42}$ This further necessitates future immunohistochemical studies to support the present findings. Similarly, the current methods used for detection of miRNA effects on target gene expression were conducted at the mRNA level. In future studies, more physiologically correct results could be achieved by examining the protein level following mRNA translation.

\section{CONCLUSION}

A total of 75 differentially expressed miRNAs were identified in atherosclerotic aortic tissues taken from mouse models. These 
tissues showed unique miRNA expression profiles that will be of interest to future researchers examining the pathophysiology of atherosclerosis. The current work applied cross-verification to confirm that SOCS3 and the SOCS3-related miRNAs, miR-30a, miR-30e and miR-19b, are potential diagnostic biomarkers with likely involvement in many of the pathways contributing to ASVD development and progression. Further investigation will, however, be required to clarify the exact roles had by specific miRNAs in the pathogenic process of ASVD. Characterization of these miRNAs provides a basis for the future development of potentially useful miRNA-based therapeutics for improved clinical diagnosis, treatment and prognosis in atherosclerosis patients.

\section{ACKNOWLEDGEMENTS}

This work was supported by grants from the National Natural Science Foundation of China (number 81070216), Heilongjiang Provincial Education Department Foundation of China (number 1155hz002), Ministry of Education 'Chunhui plan' (number Z2010007) and the Funds of the First Affiliated Hospital of Harbin Medical University (number 2009L01). Microarray experiments were performed by Kang Cheng Bio-tech, Shanghai, China.

1 Wang X, Ishimori N, Korstanje R, Rollins J, Paigen B. Identifying novel genes for atherosclerosis through mouse-human comparative genetics. Am J Hum Genet 2005; 77: 1-15.

2 Pendse AA, Arbones-Mainar JM, Johnson LA, Altenburg MK, Maeda N. Apolipoprotein E knock-out and knock-in mice: atherosclerosis, metabolic syndrome, and beyond. J Lipid Res 2009; 50 (Suppl): S178-S182.

3 Bonenfant M, Provost PR, Drolet R, Tremblay Y. Localization of type 1 17beta-hydroxysteroid dehydrogenase mRNA and protein in syncytiotrophoblasts and invasive cytotrophoblasts in the human term villi. J Endocrinol 2000; 165: 217-222.

4 Moretti F, Thermann R, Hentze MW. Mechanism of translational regulation by miR-2 from sites in the $5^{\prime}$ untranslated region or the open reading frame. RNA 2010; 16: 2493-2502.

5 Chen T, Huang Z, Wang L, Wang Y, Wu F, Meng S et al. MicroRNA-125a-5p partly regulates the inflammatory response, lipid uptake, and ORP9 expression in oxLDL-stimulated monocyte/macrophages. Cardiovasc Res 2009; 83: 131-139.

6 Ji R, Cheng Y, Yue J, Yang J, Liu X, Chen $\mathrm{H}$ et al. MicroRNA expression signature and antisense-mediated depletion reveal an essential role of MicroRNA in vascular neointimal lesion formation. Circ Res 2007; 100: 1579-1588.

7 Zhang Q, Kandic I, Kutryk MJ. Dysregulation of angiogenesis-related microRNAs in endothelial progenitor cells from patients with coronary artery disease. Biochem Biophys Res Commun 2011; 405: 42-46.

8 Huang X, Le QT, Giaccia AJ. MiR-210-micromanager of the hypoxia pathway. Trends Mol Med 2010; 16: 230-237.

9 Silvestri P, Di Russo C, Rigattieri S, Fedele S, Todaro D, Ferraiuolo G et al. MicroRNAs and ischemic heart disease: towards a better comprehension of pathogenesis, new diagnostic tools and new therapeutic targets. Recent Pat Cardiovasc Drug Discov 2009; 4: 109-118.

10 Ikeda S, Kong SW, Lu J, Bisping E, Zhang H, Allen PD et al. Altered microRNA expression in human heart disease. Physiol Genomics 2007; 31: 367-373.

11 Fang Y, Shi C, Manduchi E, Civelek M, Davies PF. MicroRNA-10a regulation of proinflammatory phenotype in athero-susceptible endothelium in vivo and in vitro. Proc Natl Acad Sci USA 2010; 107: 13450-13455.

12 Izumiya M, Tsuchiya N, Okamoto K, Nakagama H. Systematic exploration of cancer-associated microRNA through functional screening assays. Cancer Sci 2011; 102: 1615-1621.

13 Liu J, Zheng M, Tang YL, Liang XH, Yang Q. MicroRNAs an active and versatile group in cancers. Int J Oral Sci 2011; 3: 165-175.
14 Sayed D, Abdellatif M. MicroRNAs in development and disease. Physiol Rev 2011; 91: 827-887.

15 Kloosterman WP, Lagendijk AK, Ketting RF, Moulton JD, Plasterk RH. Targeted inhibition of miRNA maturation with morpholinos reveals a role for miR-375 in pancreatic islet development. PLoS Biol 2007; 5: e203.

16 Eisenberg I, Eran A, Nishino I, Moggio M, Lamperti C, Amato AA et al. Distinctive patterns of microRNA expression in primary muscular disorders. Proc Natl Acad Sci USA 2007; 104: 17016-17021.

17 Cheng Y, Ji R, Yue J, Yang J, Liu X, Chen $\mathrm{H}$ et al. MicroRNAs are aberrantly expressed in hypertrophic heart: do they play a role in cardiac hypertrophy? Am J Pathol 2007; 170: 1831-1840.

18 O'Connell RM, Rao DS, Baltimore D. microRNA regulation of inflammatory responses. Annu Rev Immunol 2012; 30: 295-312.

19 Zernecke A. MicroRNAs in the regulation of immune cell functions-implications for atherosclerotic vascular disease. Thromb Haemost 2012; 107: 626-633.

20 Luo SS, Ishibashi O, Ishikawa G, Ishikawa T, Katayama A, Mishima T et al. Human villous trophoblasts express and secrete placenta-specific microRNAs into maternal circulation via exosomes. Biol Reprod 2009. 81: 717-729.

21 Jawien J, Nastalek P, Korbut R. Mouse models of experimental atherosclerosis. J Physiol Pharmacol 2004; 55: 503-517.

22 Liao F, Andalibi A, deBeer FC, Fogelman AM, Lusis AJ. Genetic control of inflammatory gene induction and NF-kappa B-like transcription factor activation in response to an atherogenic diet in mice. J Clin Invest 1993; 91: 2572-2579.

23 Piedrahita JA, Zhang SH, Hagaman JR, Oliver PM, Maeda N. Generation of mice carrying a mutant apolipoprotein $\mathrm{E}$ gene inactivated by gene targeting in embryonic stem cells. Proc Natl Acad Sci USA 1992; 89: 4471-4475.

24 Steinberg D, Parthasarathy S, Carew TE, Khoo JC, Witztum JL. Beyond cholesterol. Modifications of low-density lipoprotein that increase its atherogenicity. N Eng/ J Med 1989; 320: 915-924.

25 Zhang SH, Reddick RL, Piedrahita JA, Maeda N. Spontaneous hypercholesterolemia and arterial lesions in mice lacking apolipoprotein $\mathrm{E}$. Science 1992; 258: 468-471.

26 Menu P, Pellegrin M, Aubert JF, Bouzourene K, Tardivel A, Mazzolai L et al. Atherosclerosis in ApoE-deficient mice progresses independently of the NLRP3 inflammasome. Cell Death Dis 2011; 2: e137.

27 Carruth LM, Demczuk S, Mizel SB. Involvement of a calpain-like protease in the processing of the murine interleukin 1 alpha precursor. J Biol Chem 1991; 266: 12162-12167.

28 Raitoharju E, Lyytikainen LP, Levula M, Oksala N, Mennander A, Tarkka M et al. miR-21, miR-210, miR-34a, and miR-146a/b are up-regulated in human atherosclerotic plaques in the Tampere Vascular Study. Atherosclerosis 2011; 219: 211-217.

29 Faraoni I, Antonetti FR, Cardone J, Bonmassar E. miR-155 gene: a typical multifunctional microRNA. Biochim Biophys Acta 2009; 1792: 497-505.

30 Huang RS, Hu GQ, Lin B, Lin ZY, Sun CC. MicroRNA-155 silencing enhances inflammatory response and lipid uptake in oxidized low-density lipoprotein-stimulated human THP-1 macrophages. J Investig Med 2010; 58: 961-967.

31 Kishida K, Funahashi T, Shimomura I. Molecular mechanisms of diabetes and atherosclerosis: role of adiponectin. Endocr Metab Immune Disord Drug Targets 2012; 12: 118-131.

32 Cheng CW, Wang HW, Chang CW, Chu HW, Chen CY, Yu JC et al. MicroRNA-30a inhibits cell migration and invasion by downregulating vimentin expression and is a potential prognostic marker in breast cancer. Breast Cancer Res Treat 2012; 134: 1081-1093.

33 Watson KE, Peters Harmel AL, Matson G. Atherosclerosis in type 2 diabetes mellitus: the role of insulin resistance. J Cardiovasc Pharmacol Ther 2003; 8: 253-260.

34 Zaragosi LE, Wdziekonski B, Brigand KL, Villageois P, Mari B, Waldmann R et al. Small RNA sequencing reveals miR-642a-3p as a novel adipocytespecific microRNA and miR-30 as a key regulator of human adipogenesis. Genome Biol 2011; 12: R64.

35 Vickers KC, Remaley AT. MicroRNAs in atherosclerosis and lipoprotein metabolism. Curr Opin Endocrinol Diabetes Obes 2010; 17: 150-155.

36 Yin R, Bao W, Xing Y, Xi T, Gou S. MiR-19b-1 inhibits angiogenesis by blocking cell cycle progression of endothelial cells. Biochem Biophys Res Commun 2012; 417: 771-776.

37 Ortiz-Munoz G, Martin-Ventura JL, Hernandez-Vargas P, Mallavia B, Lopez-Parra V, Lopez-Franco $O$ et al. Suppressors of cytokine signaling 
modulate JAK/STAT-mediated cell responses during atherosclerosis. Arterioscler Thromb Vasc Biol 2009; 29: 525-531.

38 Xu X, Wang Q, Deng B, Wang H, Dong Z, Qu X et al. Monocyte Chemoattractant Protein-1 Secreted by Decidual Stromal Cells Inhibits NK Cells Cytotoxicity by Up-Regulating Expression of SOcs3. PloS One 2012; 7: e41869.

39 Tang J, Kozaki K, Farr AG, Martin PJ, Lindahl P, Betsholtz C et al. The absence of platelet-derived growth factor- $B$ in circulating cells promotes immune and inflammatory responses in atherosclerosis-prone ApoE - - mice. Am J Pathol 2005; 167: 901-912.

40 Qiao JH, Xie PZ, Fishbein MC, Kreuzer J, Drake TA, Demer LL et al. Pathology of atheromatous lesions in inbred and genetically engineered mice. Genetic determination of arterial calcification. Arterioscler Thromb 1994; 14: 1480-1497.
41 Roselaar SE, Kakkanathu PX, Daugherty A. Lymphocyte populations in atherosclerotic lesions of apoE $-/-$ and $\mathrm{LDL}$ receptor $-/$ - mice. Decreasing density with disease progression. Atertio Thromb Vasc Biol 1996; 16: 1013-1018.

42 Qiao JH, Castellani LW, Fishbein MC, Lusis AJ. Immune-complexmediated vasculitis increases coronary artery lipid accumulation in autoimmune-prone MRL mice. Arterioscler Thromb 1993; 13: 932-943.

cc)(i)(-) This work is licensed under a Creative Commons Attribution-NonCommercial-ShareAlike 3.0 Unported License. To view a copy of this license, visit http:// creativecommons.org/licenses/by-nc-sa/3.0/ 